\title{
Cross-cultural Validation of the Brazilian Portuguese Version of the McGill Quality of Life Questionnaire
}

\author{
Sheilla Oliveira Faria and Jose Eluf-Neto* \\ Department of Preventive Medicine, São Paulo University, Brazil
}

Received: March 13, 2014; Accepted: March 14, 2014; Published: May 14, 2014

*Corresponding author: Jose Eluf Neto, Department of Preventive Medicine, São Paulo University, Av. Dr. Arnaldo 455, Sala 2216 São Paulo - SP 01246 903, Brazil, Tel: +55 (11)3061-8278,+55 (11)3061-7285; +55 (11)3061-8466; Email: jelufnet@usp.br

\begin{abstract}
Background: As quality of life (QOL) is the main focus of palliative care, evaluating QOL is a very important aspect of the care offered to patients.

Aim: To produce a Brazilian Portuguese version of the McGill Quality of Life Questionnaire adapted for the Brazilian cultural context and to assess its cross-cultural validity and reliability.

Design: The original version was translated into Brazilian Portuguese in accordance with the guidelines in the literature and then adapted to the Brazilian context by conducting interviews until we reached the final version (MQOL-Br). To assess the validity of the MQOL-Br, it was applied together with the European Organization for Research and Treatment of Cancer Quality of Life Questionnaire "Core" 30 Items, the Chronic Pain Grade - Brazil and the Karnofsky Performance Scale in interviews with 101 patients from the same population. Clinical validity was tested by conducting a known-groups comparison. Multiple linear regression analysis was used to determine which of the five subscales had independent relationships with overall QOL.The internal consistency was assessed using Cronbach's $\alpha$. Testretest reliability was assessed in a second interview with 63 patients 2-8 days after the initial interview.
\end{abstract}

Setting/Participants: Cancer patients in palliative care at the São Paulo Cancer Institute (ICESP).

Results: The instrument had good concurrent and construct validity. The multi-regression analysis indicated that the existential and physical domains had independent effects on overall quality of life. Patients with reduced performance status and those hospitalised reported lower QOL levels. The MQOL-Br demonstrated moderate to high internal consistency (Cronbach's $\alpha=0.75-0.84$ ).Temporal stability analysis showed intraclass correlation coefficient moderate to good for all subscales (except for support) and MQOL-Br total score.

Conclusion: The MQOL-Br is a valid and reliable questionnaire to assess quality of life in Brazilian palliative care patients.

Keywords: Validation studies; Quality of life; Neoplasms; Palliative care

\section{Introduction}

Recently, a gradual increase in some chronic diseases has been observed in tandem with the aging of the population. Globally, there has been a significant increase in the importance of cancer, and according to a recent report from the International Agency for Research on Cancer, its overall impact has more than doubled in the past 30 years [1]. The burden of cancer will increase to 22 million new cases each year by 2030: an increase of $75 \%$ compared with $2008,81 \%$ in low and middle Human Development Index (HDI) countries and 69\% in high and very high HDI countries [2].

The epidemiological transition in Brazil is not occurring as in most industrialized countries, but malignant neoplasms are already the second leading cause of death in the Brazilian population. In 2010, cancer deaths represented almost $17 \%$ of the deaths from known causes: 92.587 in males and 79.457 in females [3].

According to the World Health Organization, in less developed countries, like Brazil, most individuals with cancer have advanced disease at the time of diagnosis; therefore, the proportion of patients who require palliative care is enormous [4]. Palliative care is an approach that improves the quality of life of patients and their families facing problems associated with life-threatening illness through the prevention and relief of suffering [5].

Quality of life (QOL) is a multi-dimensional concept, which involves various domains such as physical, functional independence, psychological, social, spiritual, and existential wellbeing [6]. As QOL is the main focus of palliative care, assessing QOL is of paramount importance for evaluating the care offered to patients [7]. An ideal assessment of QOL should be performed using validated patient-reported outcome instruments [8].

For any instrument to be used in another country, translation and cultural adaptation are necessary to ensure equivalence between the original and translated versions [9] Validation of an instrument is also necessary to verify the extent to which the instrument measures what it is proposed to measure $[10,11]$.

In Brazil, there are few validated quality of life questionnaire. Only the European Organization for Research and Treatment of Cancer Quality of Life Questionnaire Core 15 PAL (EORTC QLQC15-PAL)and the Palliative Care Outcome Scale (POS)are specific to patients in palliative care [12]. The validation study of the POS 
has not been published yet. A statement as to why the MQOL is likely to be better, or needed would be good to include.

The McGill Quality of Life Questionnaire (MQOL) is a quality of life questionnaire that is specific for use in palliative care patients. In a recent systematic review of the existing instruments for assessing the quality of life of patients in palliative care, MQOL received top scores for its psychometric properties [7]. In addition, it has been validated in many other languages [1321]. Furthermore, other studies have reported evidence of its acceptability and psychometric properties, such as convergent and divergent validity and internal consistency, for ambulatory cancer patients and hospitalised patients in palliative care $[22,23]$

Despite all these properties and validations in several languages, MQOL has not been translated for use in Brazil. A culturally adapted and validated version of a questionnaire about the quality of life of patients in palliative care that is widely used can be helpful both for conducting clinical and epidemiological research about this topic and for clinical practice. Thus, the aims of this study were to produce a Brazilian Portuguese version of the McGill Quality of Life Questionnaire adapted to the Brazilian cultural context (MQOL-Br) and to investigate its cross-cultural validity and reliability.

\section{Methods}

\section{Participants}

All consecutive patients who met the following inclusion criteria were considered eligible for the cross-cultural adaptation study: 1) hospitalised or outpatients with an advanced stage of cancer to the Palliative Care Service of São Paulo Cancer Institute (ICESP); 2) any sex, with an age greater than 18 years; 3 ) conscious and able to communicate readily; and 4) able to provide meaningful informed consent.

São Paulo Cancer Institute (ICESP) is the largest oncology center in Latin America and is located in the complex of the "Hospital das Clínicas", Faculty of Medicine, University of São PauIo. ICESP has 580 beds (84 in Intensive Care Units).

\section{Cross-cultural adaptation study}

Permission was obtained from the original author to use the English version of the MQOL questionnaire. The cross-cultural adaptation followed the guidelines established by Beaton et al. with modifications proposed by Bracher and collegues $[9,24]$. Two independent translators, both native Brazilians, translated the MQOL from English into Brazilian Portuguese. A synthesis of the translations was agreed upon by consensus between the translators.

Ten participants were interviewed to verify their comprehension of the combined translation. The patients completed the questionnaire with assistance: the questions were read aloud by the principal investigator. The participants were asked about their understanding of the questionnaire and were invited to offer suggestions that could clarify its meaning. An expert committee reviewed all of the reports and reached consensus on pre-final version 1 . This team of five individuals included an epidemiologist, a dietician, a language professional, a chiropractor and a palliative care doctor.

Additional interviews were conducted with 40 participants. Because the participants had difficulty understanding questions 3 and 5 on pre-final version 1 , there was a second committee meeting in which it was decided to modify the wording of those questions. We sent the suggested changes to the original author to determine whether she agreed with them (pre-final version 2). This version was administered to 10 other participants. This group had no difficulties; thus, pre-final version 2 was emailed to an expert committee and the original author and was considered the final version (MQOL-Br).

Two independent native English speakers who spoke Brazilian Portuguese fluently created a back translation into English. The translators were blinded to the original version. The back translations were synthesised by consensus between the translators, and the committee and the principal author examined the combined version.

\section{Validation Study}

Study Participants: We identified 218 patients during a 3.5 month data collection period. Two duplicate patients, one who was not enrolled in the palliative care program and 27 illiterate patients were not eligible.

We excluded 48 patients who were unable to answer the questionnaire for various reasons: speech, hearing or visual deficits; confusion or sedation induced by medications; or the terminal phase of illness. Responses were received from 101 of 140 patients; thus, the participation rate was $72.1 \%$.

Interviews: The interviewers explained the study process and obtained voluntary agreement from the patients through written consent.

For the validation study, outsourced interviewers were hired. All of the interviewers underwent training, and received an interviewer's manual. The patients completed the questionnaires with assistance: the questions were read aloud by the interviewers.

\section{Instruments}

McGill Quality of Life Questionnaire (MQOL): The MQOL was developed specifically to measure the QOL of patients facing a life-threatening illness using five subscales: physical symptoms, physical well-being, psychological well-being, existential wellbeing and support [25].The MQOL consists of 16 items assessed on a scale of 0 to 10 and a single-item rating scale (SIS) constructed to measure global QOL [26] Studies have shown that the MQOL is valid, reliable and acceptable for measuring the QOL of cancer patients and that it has good responsiveness [22, 23, 27].

European Organization for Research and Treatment of Cancer Quality of Life Questionnaire "Core" 30 Items (EORTC QLQ-C30): The EORTC QLQ-C30 is a 30-item, cancerspecific questionnaire for assessing the health-related QOL of cancer patients, although it is not specific to palliative care. The 
EORTC QLQ-C30 is divided into five functional scales (physical, cognitive, emotional, social and role), three symptom scales (fatigue, pain, nausea and vomiting) and a global health and QOL scale; it also contains six other items that assess symptoms commonly reported by cancer patients (dyspnoea, appetite loss, sleep disturbance, constipation and diarrhoea) and the perceived financial impact of the disease and treatment [28] In a Brazilian study conducted with a sample of cancer patients, the EORTC QLQ-C30 showed good reliability based on a measurement of its internal consistency using Cronbach's $\alpha$, except for the items related to cognitive functioning [29].

Karnofsky Performance Scale (KPS): The KPS classifies patients according to their degree of functional impairment on a scale from 0 to 100 . This scale assesses the patient's ability to perform activities of daily living and work activities and his or her need for special care [30]. A study conducted by Mor et al. demonstrated the reliability and validity of the KPS scale [31].

Chronic Pain Grade - Brazil (CPG-Br): The CPG-Br is an eight-item questionnaire that recalls aspects of the most bothersome pain experienced in the last 3 or 6 months. Pain frequency, intensity and interference with usual activities are assessed. The CPG was adapted to the Brazilian cultural context (CPG-Br) [24] They found good internal consistency assessed by Cronbach's $\alpha$, and the test-retest reliability was considered adequate. The correlations identified between the CPG-Br and the scales used for comparison indicated that the CPG-Br was valid in the target population.

\section{Data analysis}

Stata ${ }^{\circledR} 10.0$ (Stata Corp. 2007. College Station, TX: US) for Windows was used for the data analysis. The socio-demographic data and the scores were analysed descriptively and presented as frequencies. We calculated Pearson correlation coefficients for normally distributed variables and Spearman coefficients for variables that were non-normally distributed.

This project was approved by the ICESP Research Center and the Research Ethics Committee of the Faculty of Medicine, São Paulo University (Research protocol $n^{\circ} 400 / 10$ ).

\section{Validity}

Concurrent validity was assessed by calculating the correlation coefficient between the total scores on the MQOL-Br and the EORTC QLQ-C30, and construct validity was assessed by calculating the correlation coefficients between the MQOL$\mathrm{Br}$, the KPS and the CPG-Br. Convergent and divergent validity were checked by measuring the extent to which the MQOL-Br subscales were correlated with similar and different constructs on the EORTC QLQ-C30 functioning scales and the classification of pain on the CPG-Br.

We evaluated the clinical validity of the MQOL-Br using a known-groups comparison. In other words, we evaluated the extent to which the questionnaire scores differentiated patients according to their performance status (KPS) and the type of care (outpatient or hospitalised). We hypothesised that patients with poor performance status and hospitalised patients would report worse QOL.

Furthermore, to determine which of the five subscales had independent relationships with overall quality of life, we performed a multiple linear regression analysis in which SIS was entered as a dependent variable and the MQOL-Br subscales were entered as independent variables. The same regression was performed excluding the physical well-being subscale.

Cronbach's $\alpha$ coefficient was calculated to assess the internal consistency of the questionnaire.The test-retest reliability of the MQOL-Br was assessed by comparing the MQOL-Br scores obtained from the same participant at two different times. Patients were asked to respond to the MQOL-Br a second time by telephone approximately 2-3 days after the initial interview, with a maximum interval of 7 days. Sixty-three patients were interviewed a second time. The main reason for not completing the retest was difficulty making telephone contact (51.1\%), followed by the clinical deterioration of the patient $(16.2 \%)$ and hospitalisation at another institution (13.5\%). The MQOL-Br subscale and total scores obtained on these two occasions were compared using intra-class correlation coefficients.

\section{Results}

\section{Cross-cultural adaptation study}

Sixty patients were interviewed during an 8-month period. Most of the participants were male (57.4\%), and the mean age was $60.1(\mathrm{SD}=14.9)$ years.

Thirty-five percent of our patients were unable to understand item three (3) of the heading of the Part B. They did not understand where to write "none" and where to write the symptoms. Thus, item (3) was expanded with more detailed instructions:

"(3) If you have had three (3) symptoms or physical problems in the last two (2) days, answer the first three questions. For each question, write a symptom or physical problem on the line and circle the number that best shows the importance of each one.

If you have had only two (2) symptoms or physical problems in the last two (2) days, write them on the line for the first two questions and circle the number that best shows the importance of each one. Write "none" on the line of the last question and do not circle a number.

If you have had only one (1) symptom or physical problem in the last two (2) days, write them on the line for the first question and circle the number that best shows the importance of this symptom. Write "none" on the line for questions 2 and 3 and do not circle a number.

If you have had no symptoms or physical problems in the last two (2) days write "none" on the line of each question and do not circle a number."

As some participants (15\%) could not understand the concept of "depressed", on question 5, two words -run down, and listless were added (between brackets) - to help understanding. We sent the suggested changes to the original author and she agreed with them. The final adapted version, called McGill Quality of 
Life Questionnaire for Brazil (MQOL-Br), was approved by the original author.

\section{Validation study}

The mean age was $62(\mathrm{SD}=15.3)$ years, and the proportions of males and females were almost equal. Most of the patients had poor functional status (KPS $\leq 60$ ) and incomplete high school education (Table 1).The most frequently listed symptoms were pain $(70.3 \%)$, weakness $(19.8 \%)$ and tiredness $(12.9 \%)$.

The data for the subscales and each item of the MQOL-Br are shown in Table 2. The support subscale had the highest mean score, and the physical symptoms subscale had the lowest mean score.

We found that the MQOL-Br total score was positively correlated with global health status/QOL on the EORTC QLQ-C30 $(\mathrm{r}=0.69, \mathrm{p}<0.01)$.The MQOL-Br total score was negatively correlated with the pain classification on the CPG-Br ( $r=-0.29$, $p$ $=0.01)$ and positively correlated with the KPS $(r=0.22, p=0.03)$.

Table 3 presents the correlations between the MQOL subscales, the functional scales and the global health status/ QOL item on the EORTC QLQ-C30 and the classification of pain on the CPG-Br. Only the well-being subscale correlated positively with global health status/QOL on the EORTC QLQ-C30. The correlations between the MQOL-Br subscales and the EORTC QLQ-C30 scales ranged from poor to high.

Patients with gravely reduced performance status (KPS $\leq 60)$ and hospitalised patients reported lower QOL levels than patients with good status and outpatients. However, there were only significant differences in the existential subscale score and the total score between the KPS groups and in the physical symptoms

Table 1: Socio-demographic and Clinical Characteristics of the Study Sample $(\mathrm{n}=101)$.

\begin{tabular}{|c|c|c|}
\hline Characteristics & & n (\%) \\
\hline \multirow{3}{*}{ Age, years } & $<45$ & $13(12.9)$ \\
\hline & $45-64$ & $43(42.6)$ \\
\hline & $\geq 65$ & $45(44.5)$ \\
\hline \multirow{2}{*}{ Gender } & Male & $51(50.5)$ \\
\hline & Female & $50(49.5)$ \\
\hline \multirow{2}{*}{ Education } & $<$ High school graduation & $78(77.2)$ \\
\hline & $\geq$ High school graduation & $23(22.8)$ \\
\hline \multirow{3}{*}{ Religion } & Catholic & $57(56.4)$ \\
\hline & Evangelical & 30 (29.7) \\
\hline & Others & $14(13.9)$ \\
\hline \multirow{2}{*}{ Marital status } & Married & $52(51.5)$ \\
\hline & Single & $49(48.5)$ \\
\hline \multirow{2}{*}{ KPS } & $\leq 60$ & $59(58.4)$ \\
\hline & $>60$ & $42(41.6)$ \\
\hline \multirow{4}{*}{ Cancer site } & Colon & $12(12.0)$ \\
\hline & Lung & $10(9.9)$ \\
\hline & Breast & $9(8.9)$ \\
\hline & Others & $70(69.2)$ \\
\hline
\end{tabular}

Note. KPS: Karnofsky Performance Scale
Table 2: Descriptive Statistics for the McGill Quality of Life QuestionnaireBrazil Subscales and Items, the SIS and the Total Score.

\begin{tabular}{|c|c|}
\hline Global QOL scores and items ${ }^{a}$ & Mean (SD) \\
\hline SIS & $6.1(2.5)$ \\
\hline Physical symptoms & $5.6(3.3)$ \\
\hline 1) First physical problem & $3.6(3.6)$ \\
\hline 2) Second physical problem & $5.8(4.1)$ \\
\hline 3) Third physical problem & $7.4(4.0)$ \\
\hline Physical well-being & $5.7(3.0)$ \\
\hline 4) Physically well/terrible & $5.7(3.0)$ \\
\hline Psychological symptoms ${ }^{\mathrm{a}}$ & $5.7(3.4)$ \\
\hline 5) Depressed & $6.0(3.9)$ \\
\hline 6) Nervous and worried & $5.4(4.0)$ \\
\hline 7) Sad & $5.7(3.9)$ \\
\hline 8) Fearful of the future & $5.9(3.7)$ \\
\hline Existential well-being ${ }^{\mathrm{b}}$ & $7.3(2.2)$ \\
\hline 9) Existence is meaningful & $6.7(3.4)$ \\
\hline 10) Achieving goals & $6.7(3.0)$ \\
\hline 11) Life is worthless & $8.2(2.5)$ \\
\hline 12) Control over time & $7.0(3.2)$ \\
\hline 13) Like self & $7.6(2.9)$ \\
\hline 14) Every day is a gift & $7.4(3.1)$ \\
\hline Support $^{\mathrm{c}}$ & $8.5(1.8)$ \\
\hline 15) World is caring & $7.8(2.7)$ \\
\hline 16) Feel supported & $9.2(1.9)$ \\
\hline Total Score ${ }^{\mathrm{d}}$ & $6.6(2.1)$ \\
\hline
\end{tabular}

Notes.QOL $=$ quality of life; SIS = single-item scale. ${ }^{\text {a }} 1$ missing. Scores ranged from 0 to 10 except as indicated: ${ }^{\mathrm{b}} 0.5$ to 10 ; ${ }^{\mathrm{c}} 2.5$ to 10 ; ${ }^{\mathrm{d}} 2.2$ to 10 .

subscale score between the types of care groups (Table 4).

Only the physical well-being subscale showed a statistically significant relationship with overall quality of life (SIS). When this subscale was excluded from the regression analysis, the physical symptoms subscale and the existential subscale had statistically significant relationships with SIS (Table 5).

\section{Reliability}

Cronbach's $\alpha$, the indicator of internal consistency reliability, was moderate to high for all of the MQOL subscales and the total score: physical symptoms $=0.76$; psychological symptoms $=0.75$; existential well-being $=0.77$; support $=0.84$; and total MQOL $=$ 0.82 .Based on the analysis of temporal stability, the intraclass correlation coefficient (ICC) was moderate to good for the total score on the MQOL-Br and all of the subscales (except for support, which had a poor ICC) (Table 6).

\section{Discussion}

This study demonstrated that the MQOL-Br is a valid, reliable and acceptable measure for assessing QOL in Brazilian cancer patients in palliative care. 
Table 3: Correlations Between the EORTC QLQ-C30 (Global Health Status/QOL and Functioning Scales), the CPG-Br and the McGill Quality of Life Questionnaire-Brazil (MQOL-Br).

\begin{tabular}{|l|c|c|c|c|c|}
\hline & \multicolumn{3}{|c|}{ MQOL-Br Subscales } \\
\hline EORTC QLQ-C30 & Physical symptoms & Physical well-being & Psychological symptoms & Existential well-being & Support \\
\hline Global health status/QOL & 0.10 & $0.40^{*}$ & 0.07 & 0.24 \\
\hline Functional scales & & & 0.05 \\
\hline Physical functioning & 0.15 & $0.22^{\mathrm{b}}$ & -0.02 & -0.09 \\
\hline Role functioning & 0.12 & 0.09 & 0.09 & -0.03 & $0.20^{\mathrm{b}}$ \\
\hline Emotional functioning & $0.56^{\mathrm{a}}$ & $0.45^{\mathrm{a}}$ & $0.79^{\mathrm{a}}$ & $0.52^{\mathrm{a}}$ & 0.09 \\
\hline Cognitive functioning & -0.03 & -0.01 & -0.03 & 0.16 & 0.07 \\
\hline Social functioning & 0.07 & -0.13 & -0.12 & 0.20 & $0.20^{\mathrm{a}}$ \\
\hline CPG-Br & $-0.28^{\mathrm{b}}$ & $-0.38^{\mathrm{a}}$ & -0.22 & -0.15 & -0.14 \\
\hline
\end{tabular}

Notes.EORTC QLQ-C30: European Organization for Research and Treatment of Cancer Quality of Life Questionnaire "Core" 30 Items. CPG-Br: Chronic Pain Grade - Brazil. ${ }^{\mathrm{a}} \mathrm{p}<0.01{ }^{\mathrm{b}} \mathrm{p}<0.05$

Table 4: MQOL-Br Differentiation of Karnofsky Performance Status and Type of Care.

\begin{tabular}{|l|c|c|c|c|c|c|}
\hline & \multirow{2}{*}{$\begin{array}{c}\text { MQOL-Br, mean } \\
\text { (SD) }\end{array}$} & $\begin{array}{c}\text { Physical } \\
\text { symptoms }\end{array}$ & $\begin{array}{c}\text { Physical well- } \\
\text { being }\end{array}$ & $\begin{array}{c}\text { Psychological } \\
\text { symptoms }\end{array}$ & $\begin{array}{c}\text { Existential well- } \\
\text { being }\end{array}$ & Support \\
\cline { 5 - 7 } & & & & & & \\
\hline Performance & $6.19(2.17)$ & $5.11(3.61)$ & $5.15(3.09)$ & $5.35(3.33)$ & $6.90(2.29)$ & $8.42(1.81)$ \\
\hline KPS $\leq 60$ & $7.10(1.99)^{\mathrm{a}}$ & $6.28(2.69)$ & $6.40(2.59)^{\mathrm{a}}$ & $6.30(3.36)$ & $7.89(1.99)^{\mathrm{a}}$ & $8.62(1.89)$ \\
\hline KPS > 60 & & & & & & \\
\hline Type of care & $5.73(1.87)$ & $4.00(3.16)$ & $5.06(2.94)$ & $4.66(3.39)$ & $6.63(2.46)$ & $8.29(1.48)$ \\
\hline Hospitalised & $6.74(2.16)$ & $5.92(3.25)^{\mathrm{a}}$ & $5.79(2.95)$ & $5.97(3.33)$ & $7.46(2.15)$ & $8.55(1.91)$ \\
\hline Outpatient & &
\end{tabular}

Notes.KPS: Karnofsky Performance Scale. MQOL-Br: McGill Quality of Life Questionnaire-Brazil. ${ }^{a} \mathrm{p}<0.05$

Table 5: Beta Coefficients of the McGill Quality of Life Questionnaire (MQOL-Br) Subscales in the Multiple Regression Analysis with the Single-item Scale as the Dependent Variable, with and without the Physical Well-being Subscale.

\begin{tabular}{|l|l|l|l|l|l|}
\hline \multicolumn{5}{|l|}{ MQOL-Br subscales } & \multicolumn{3}{l|}{} \\
\hline Physical symptoms & Physical well-being & Psychological symptoms & Existential well-being & Support \\
\hline 0.05 & $0.39^{\text {a }}$ & -0.02 & 0.23 & 0.16 \\
\hline Without physical well-being & Physical well-being & Psychological symptoms & Existential well-being & Support \\
\hline Physical symptoms & -- & 0.00 & $0.37^{\text {a }}$ & 0.13 \\
\hline $0.21^{\text {a }}$ & & & \\
\hline
\end{tabular}

Note. ${ }^{\mathrm{a}} \mathrm{p}<0.01$

The modifications proposed by Bracher and colleagues [24] for the adaptation study was extremely important for this process to be successful, because: (1) interviews to check comprehension enabled the committee to know the problems of understanding of the patients and then propose the necessary modifications, (2) back-translations were made from the final version, and therefore incorporated all the changes of the process of cultural adaptation, (3) the realization of two back translations and their synthesis enable to get a more accurate and higher equivalence with the original version.

Our study demonstrates that MQOL-Br has good convergent and divergent validity. The correlation observed between the MQOL-Br subscales and the classification of pain on the CPG-Br supports the convergent validity of the MQOL-Br because the physical dimensions of the MQOL-Br are aligned conceptually with the CPG-Br. The correlations between the MQOL-Br
Table 6: Intraclass Correlation Coefficients (ICC) between the McGill Quality of Life Questionnaire (MQOL-Br) Scores on the Test and Retest $(n=63)$.

\begin{tabular}{|l|c|c|}
\hline MQOL-Br Subscales & ICC & {$[95 \% \mathrm{CI}]$} \\
\hline Physical symptoms & 0.61 & $0.42-0.74$ \\
\hline Physical well-being & 0.53 & $0.33-0.69$ \\
\hline Psychological symptoms & 0.65 & $0.48-0.77$ \\
\hline Existential well-being & 0.66 & $0.49-0.78$ \\
\hline Support & 0.37 & $0.14-0.57$ \\
\hline Total score & 0.72 & $0.57-0.82$ \\
\hline
\end{tabular}

Note. $\mathrm{p}<0.001$, except support $(\mathrm{p}=0.001)$ 
subscales and the EORTC QLQ-C30 scales, although they were weak, supported the convergent and divergent validity of the MQOL-Br. When there was conceptual alignment between the dimensions, the correlation was almost always statistically significant. Conversely, when the scales measured different constructs, the correlation was not statistically significant.

Our regression findings corroborate the literature with regard to the importance of existential well-being and symptom control for patients with incurable cancer [22, 32]. Good testretest reliability of the MQOL-Br, with moderate to good intra class correlation coefficients, was found.

This paper had some limitations. First, the test-retest sample was small. One of the biggest problems facing researchers studying the quality of life of seriously ill people is the high nonresponse rate. Second, as our study was performed with terminal cancer patients, the results may not be generalizable to other patients with other chronic disease.

As reported in other studies, the subscale on the MQOL$\mathrm{Br}$ with the worst average score was the physical symptoms subscale, and the support subscale had the best average score $[13,15,21-23]$. The most common symptoms identified were consistent with those reported in the literature [14,33-35].

Some differences were found between our study and others MQOL studies. The Global Quality of Life (SIS) score and the MQOL-Br total score were lower than the corresponding scores reported in the validation studies conducted in Canada and Iran $[18,22]$. The SIS score was higher than the scores reported in MQOL studies in Israel and Japan $[13,21]$. The likely explanation for these findings is related to the types of patients who were included in each study.

The correlations between the MQOL-Br and the EORTCQLQ C30 revealed different results in the Korea study. A likely explanation is the higher education of Korean patients, and thus participants may have shown greater understanding of the studied scales [15].

All of the subscales and the MQOL-Br total score showed good internal consistency (Cronbach's $\alpha>0.70$ ), unlike other validation studies that reported at least one subscale with an $\alpha<0.70$ [1321].

Few studies verifying test-retest reliability of the MQOL found higher correlations between the interviews. A likely explanation is that in these studies patients were interviewed twice, face to face $[16,19]$. Because the health condition of our patients was delicate and returning to hospital would take too long, we offered the option for a telephone interview. Most of the patients were likely unable to complete the questionnaire themselves; they needed help reading and completing the items, which may have influenced their responses. In addition, it is important to remember that test-retest reliability assumes that the patient's state of health is stable at both testing times [10]. Changes in clinical status for patients in palliative care can occur in a few days and thus, the results could be different anyway.
Finally, as the palliative care concept is expansive to patients with life-threatening illnesses(both cancer and no cancer), a larger study with both types of patients will be needed, in further confirmation and evaluation of the MQOL-Br properties. In the same way, as the MQOL-Br was not applied in debilitated patients who could not complete the questionnaire, further research is needed in addressing the QOL issue in those patients.

\section{Conclusions}

The culturally adapted version of the McGill Quality of Life Questionnaire in Brazilian Portuguese demonstrated good reliability and validity when applied to cancer patients in palliative care. This questionnaire will be a useful tool for evaluating QOL in Brazilian palliative care patients.

\section{Acknowledgements}

This work was supported by the "Fundação de Amparo à Pesquisa do Estado de São Paulo (FAPESP)" [grant number 2011/52104-9].

We would like to thank Dr Robin Cohen for allowing us to translate the MQOL.

\section{References}

1. International Agency for Research on Cancer-IARC (2009) World Cancer Report 2008 Lyon: International Agency for Research on Cancer.

2. International Agency for Research on Cancer -IARC (2012).World Cancer Factsheet. London: Cancer Research UK.

3. “Ministry of Health”(2010). Department of Information and Computing SUS. Of mortality information system: proportional mortality for different causes, Brazil 1(2).

4. World Health Organization WHO (2007) Palliative Care - Cancer control: knowledge into action: WHO guide for effective programmes; module 5, Geneva, Switzerland.

5. World Health Organization(WHO) (2002) National cancer control programmes: policies and managerial guidelines ( $2^{\text {nd }}$ edn), Geneva, Switzerland.

6. Osoba D (2011) Health-related quality of life and cancer clinical trials. Ther Adv Med Oncol 3(2):57-71.

7. Albers G, Echteld MA, de Vet HCW, van der Linden MHM,Deliens L (2010) Evaluation of quality-of-life measures for use in palliative care: a systematic review. Palliat Med 24(1):17-37.

8. Paiva CE, Barroso EM, Carneseca EC, Souza CP, Santos FT, López RVM et al. (2014) A critical analysis of test-retest reliability in instrument validation studies of cancer patients under palliative care: a systematic review. BMC Med Res Methodol 14(8): 1-10.

9. Beaton DE, Bombardier C, Guimellin F, Ferraz MB (2000) Guidelines for the process of cross-cultural adaptation of self-reported measures.Spine 25(24):3186-3191

10. Lohr KN, Aaronson NK, Alonso J, Burnam MA, Patrick DL, Perrin EB et al. (1996) Evaluating quality-of-life and health status instruments: development of scientific review criteria. Clin Ther18(5):979-992.

11. Fletcher RW, Fletcher SW (2005) Clinical Epidemiology. (4 $4^{\text {nd }}$ edn), Philadelphia. Lippincott Willians \& Wilkins.

12. Nunes NA (2014) The quality of life of Brazilian patients in palliative care: 
validation of the European Organization for Research and Treatment of Cancer Quality of Life Questionnaire Core 15 PAL (EORTC QLQ-C15PAL). Support Care Cancer 22(6):1595-600.

13. Bentur N, Resnizky S (2005) Validation of the McGill Quality of Life Questionnaire in home hospice settings in Israel.Palliat Med 19(7):538 544

14. Hu WY, Dai Y.T, Berry D, Chiu T.Y (2003) Psychometric testing of the translated McGill Quality of Life questionnaire-Taiwan version in patients with terminal cancer. J Formos Med Assoc 102(2):97-104.

15. Kim SH, Ku S, Yun YH, Lee CG, Choi YS, et al. (2007) Validation study of the Korean version of the McGill Quality of Life Questionnaire. Palliat Med 21(5):441-447.

16. Lo RSK, Woo J, Zhoc KC, Li CY, Yeo W, Johnson P, et al. (2001) Crosscultural validation of the McGill Quality of Life questionnaire in Hong Kong Chinese. Palliat Med 15(5):387-397.

17. Lua PL, Wong SY (2012) The Reliability of the Malay Versions of Hospital Anxiety Depression Scale (HADS) and Mcgill Quality of Life Questionnaire (MQOL) among a Group of Patients with Cancer in Malaysia. The Malaysian Journal of Psychiatry 2(1):1-13.

18. Tolentino VR, Sulmasy DP (2002) A Spanish version of the McGill quality of life questionnaire. J Palliat Med 18(2):92-96

19. Shahidi J, Khodabakhshi R, Gohari MR, Yahyazadeh H, Shahidi, N (2008) McGill Quality of Life Questionnaire: Reliability and Validity of the Persian Version in Iranian Patients with Advanced Cancer. J Palliat Med 11(4):621-626.

20. Sguazzin C, Giorgi I, Alesii A, Fini M (2010).Italian validation of the McGill Quality of life Questionnaire (MQOL-It).G Ital Med Lav Ergon 32(3 Suppl B):B58-62.

21. Tsujikawa M, Yokoyama K, Urakawa K, Onishi K (2009) Reliability and Validity of the Japanese Version of the McGill Quality of Life Questionnaire assessed by application in palliative care wards. Palliat Med 23(7):659-664.

22. Cohen SR, Mount BM, Tomas J, Mount L (1996) Existential well-being is an important determinant of quality of life: evidence from the McGill Quality of life Questionnaire. Cancer 77(3):576-586.

23. Cohen SR, Mount BM, Bruera E, Provost M, Rowe J et al. (1997) Validity of the McGill Quality of Life Questionnaire in the palliative care setting a multi-centre Canadian Study demonstrating the importance of the existential domain. Palliat Med 11(1):3-20.
24. Bracher ESB, Pietrobon, R, Eluf-Neto J (2010) Cross-cultural adaptation and validation of a Brazilian Portuguese version of the chronic pain grade. Qual Life Res19(6):847-852.

25. Cohen SR, Mount BM, Strobel MG, Bui F (1995) The McGill Quality of Life Questionnaire: a measure of quality of life appropriate for people with advanced disease. A preliminary study of validity and acceptability. Palliat Med 9(3):207-219.

26. Henry M, Huang LN, Ferland MK, Mitchell J, Cohen SR (2008) Continued study of the psychometric properties of the McGill quality of life questionnaire. Palliat Med 22(6):718-723

27. Cohen SR, Mount BM (2000) Living with cancer: "Good" days and "Bad" days- What produce them? Cancer 89(8):1854-1865.

28. Aaronson NK, Ahmedzai S, Bergman B, Bullinger M, Cull A, Duez NJ et al. (1993) The European Organization for Research and Treatment of Cancer QLQ-C30: a quality-of-life instrument for use in international clinical trials in oncology. J Natl Cancer Inst 85(5):365-376.

29. Ferreira KSL, Teixeira MJ, Mendonza TR, Cleeland C S (2010).Validation of brief pain inventory to Brazilian patients with pain. Support Care Cancer 19(4):505-11.

30. Shag CC, Heinrich RL, Ganz PA (1984) Karnofsky performance status revisited: reliability, validity, and guidelines. J Clin Oncol 2(3):187-193.

31. Mor V, Laliberte L, Morris JN \& Wiemann MI (1984) The Karnofsky Performance Status Scale-An Examination of its Reliability and Validity in a Research Setting. Cancer 53(9):2002-2007.

32. Chang VT, Hwang SS, Feuerman M, Kasimis BS (2000) Symptom and quality of life survey of medical oncology patients at a veterans affairs medical center: a role for symptom assessment. Cancer 88(5):1175-1183.

33. Teunissen SCCM, Wesker W, Kruitwagen C, de Haes HCJM, Voest EE et al. (2007) Symptom Prevalence in Patients with Incurable Cancer: A Systematic Review. J Pain Symptom Manag 34 (1):94-104.

34. Spichiger E, Müller-Fröhlich C, Denhaerynck K, Stoll H, Hantikainen V, Dodd, M (2011) Symptom prevalence and changes of symptoms over ten days in hospitalized patients with advanced cancer: A descriptive study. Eur J OncolNur 15(2):95-102.

35. Kirkova J, Rybicki L, Walsh D, Aktas A, Davis MP, Karafa MT (2011) The relationship between symptom prevalence and severity and cancer primary site in 796 patients with advanced cancer. Am J Hosp Palliat Care 28(5):350-355. 